\title{
Real-time Composition Determination of Gas Mixtures
}

\author{
J.C. Lötters ${ }^{1,2}$, E.J. van der Wouden ${ }^{1}$, J. Groenesteijn ${ }^{2}$ \\ W. Sparreboom ${ }^{1}$, T.S.J. Lammerink ${ }^{2}$, R.J. Wiegerink ${ }^{2}$ \\ j.c.lotters@bronkhorst.com; j.c.lotters@utwente.nl \\ ${ }^{1}$ Department of Research \& Development, Bronkhorst High-Tech BV, Ruurlo, The Netherlands \\ ${ }^{2}$ MESA+ Institute for Nanotechnology, University of Twente, Enschede, The Netherlands
}

\begin{abstract}
We have designed and implemented an analytical calculation model with which we can real-time determine the composition of gas mixtures. The model is based upon a multi-parameter flow measurement system, consisting of a Coriolis and thermal flow sensor, a density meter and a pressure sensor. The system enables direct measurement of flow rate and physical properties of gas mixtures, including density, viscosity and heat capacity. We demonstrated real-time composition determination of binary and ternary gas mixtures. For fuel gases, we demonstrated real-time determination of the energy content (Wobbe index) of the gas mixture from its composition and density.
\end{abstract}

Key words: composition determination; gas mixtures; multiparameter measurement; Coriolis flow sensor; thermal flow sensor

\section{Introduction}

It is expected that the composition and quality of the natural gas in national gas grids is going to vary enormously due to the blending of natural gas from different countries, the addition of biogas and periodical variations in both gas types. Moreover, the introduction of biogas in national gas grids is hindered by the absence of a cheap, reliable and miniature device for the energy content measurement of fuel gases. The multi-parameter flow measurement system, as presented in this paper, will provide a point-of-use solution for these and other applications, e.g. burner control, where constant gas quality is required. Other applications are for instance quality control in medical gas mixtures, and oxygen or hydrogen detection in semiconductor equipment.

At the moment, only conventional, large and expensive equipment exists for the energy content measurement of fuel gases, e.g. a Wobbe index meter [1] or a gas chromatograph [2]. Some recent developments towards miniaturised equipment are based on correlations between the Wobbe Index [3] and specific parameters of the gas, such as the viscosity [4] or thermal conductivity [5]. However, as we will show in this paper, methods relying on only one parameter are not sufficient and potentially lead to very large errors in the Wobbe index.
In previous work [6], we presented the hardware and the basic model to determine the flow and the physical properties of single gases provided. In this work, we present an extended analytical model that enables calculation of the composition and the energy content of binary and ternary gas mixtures.

\section{Basic System Structure}

The basic structure of the multi-parameter flow measurement system is shown in figure 1 . The system consists of a Coriolis and thermal flow sensor, a density meter and a differential pressure sensor. Gas flow enters the system at the inlet, passes through the density meter, the Coriolis and the thermal flow sensor, and leaves the system at the outlet. The differential pressure between the inlet and outlet of the thermal flow sensor is measured by the pressure sensor.

\section{Operating principle}

The output signal of the thermal flow sensor is a measure for the flow rate and the heat capacity of the gas mixture. The pressure drop over the thermal flow sensor is measured by the differential pressure sensor. The output signal of the Coriolis mass flow sensor provides the mass flow, and the density is obtained from the density sensor. 


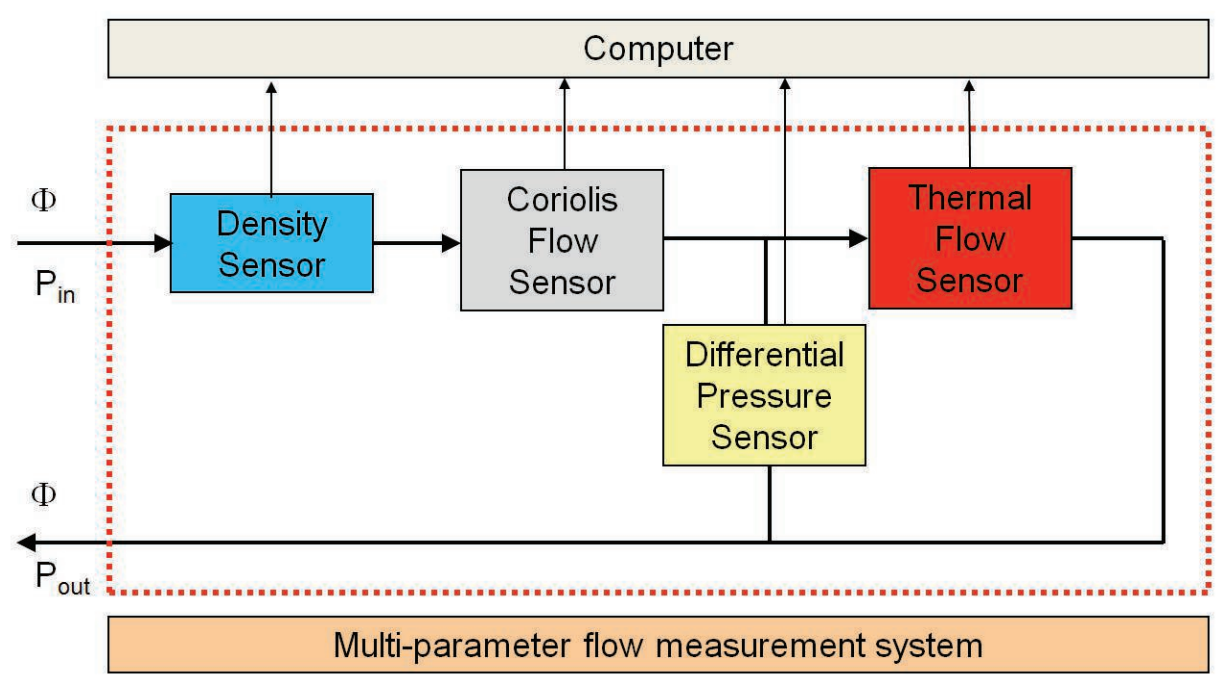

Fig. 1: Basic structure of the multi-parameter flow measurement system

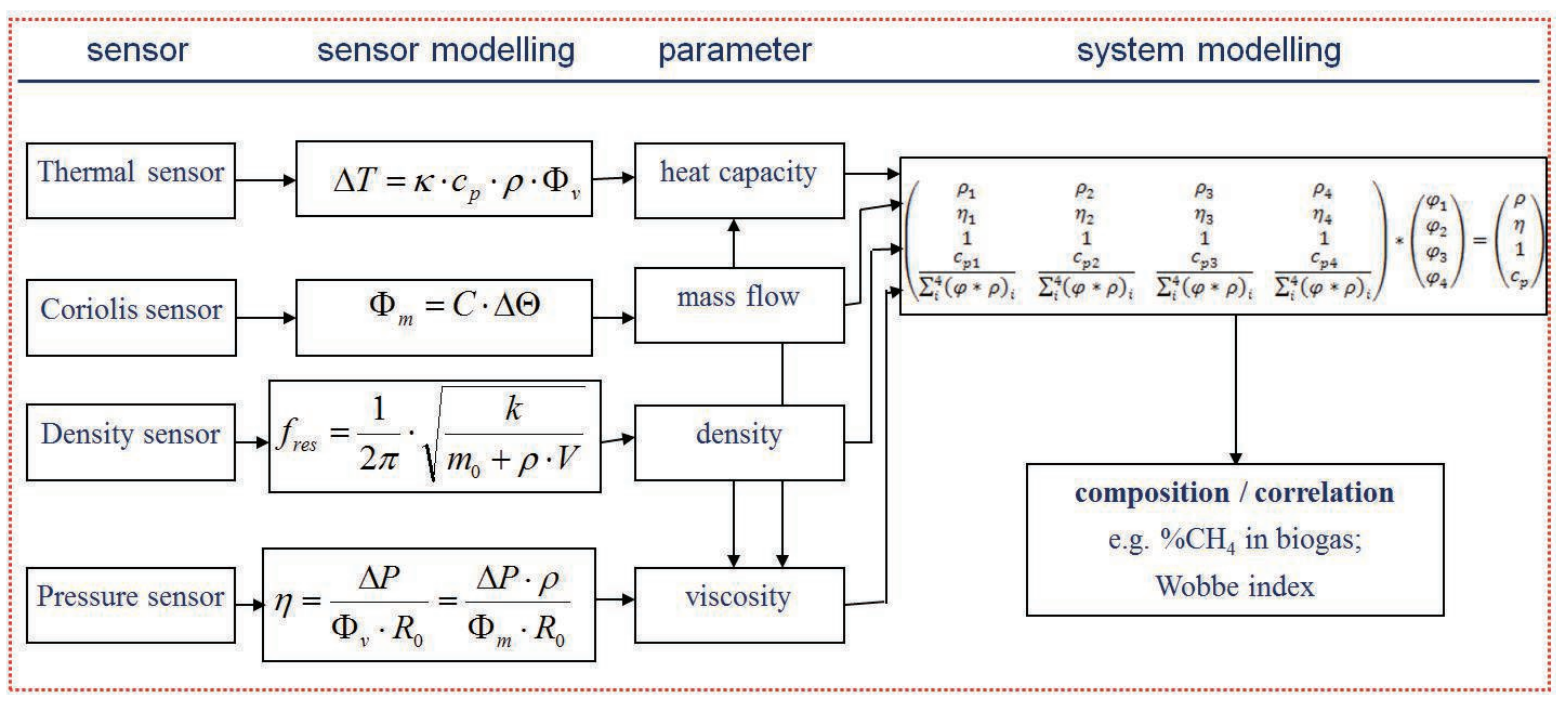

Fig. 2: Analytical calculation model for real-time gas mixture composition determination

As shown in figure 2, the other parameters of the gas mixture can be obtained from the output signals via a calculation model. By comparing the output signals of the Coriolis flow sensor and the pressure sensor, and taking the density into account, the viscosity of the medium can be calculated. By comparing the output signals of the thermal and the Coriolis flow sensors, the heat capacity of the medium can be determined.

Thus, the different parameter values of the mixture can be obtained. Additionally, we know the sum of all fractions of the gases present in the mixture equals $100 \%$. Subsequently, when we know the individual gases present in the mixture, we can calculate the composition of the gas mixture.

\section{Modelling}

In [6], we explained how the different physical parameters of a gas mixture are derived from the sensor signals. This is the part in figure 2 indicated as "sensor modelling".

The part in figure 2 labelled "system modelling" shows the mathematical model that we constructed to relate the measured parameters to fractions of a gas mixture. In the formula, the left hand side is a parameter matrix that represents the physical parameters of each individual gas. The second column represents the volume fractions of the mixture. The product of the two gives the measured physical parameters. 
Now, since we know the sum of all fractions of the gases present in the mixture equals $100 \%$, the individual gases that are present in the mixture, and the different parameter values of the mixture, we can solve the matrix equation by modifying the volume fractions of the mixture according to the least squares fitting method. Thus, we obtain the fractions of the individual gases, and therefore, we know the composition of the gas mixture.

With the current version of the system model, we are able to determine the composition of gas mixtures consisting of 3 or 4 different individual gases. The model can be extended to 5 gases, e.g. by adding the thermal conductivity. The fraction of each individual gas in a binary gas mixture can be calculated using a linear equation, e.g. based on the heat capacity.

The Wobbe Index $\mathrm{WI}\left[\mathrm{MJ} / \mathrm{Nm}^{3}\right]$ can be calculated with [1]:

$$
W I=\frac{H}{\sqrt{G_{s}}}
$$

where $\mathrm{H}\left[\mathrm{MJ} / \mathrm{Nm}^{3}\right]$ is the amount of heat generated by the complete combustion of a certain volume of a gas mixture with air; and $\mathrm{G}_{\mathrm{s}}$ [-] is the ratio of the mass densities of the gas mixture and air. Since we know the composition of the mixture, the Wobbe Index can be determined by taking into account the respective heating value of each fraction and the relative mass density of the gas mixture.

\section{Simulation results}

The composition and Wobbe index of the natural gas provided to the Dutch national gas grid is varying quite a lot due to the blending of natural gas from different countries, the addition of biogas and periodical variations in both gas types [7].

In figure 3, the variation in the Wobbe index is shown of a gas mixture resembling biogas consisting of $\mathrm{CH}_{4}, \mathrm{CO}_{2}$ and $\mathrm{N}_{2}$. The Wobbe index depends differently on the nitrogen and carbon dioxide fraction.

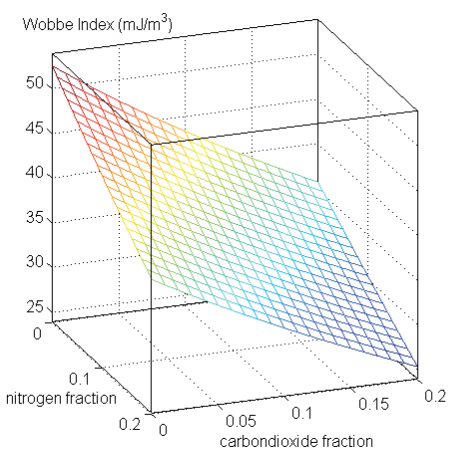

Fig. 3: Dependency of the Wobbe index on the $\mathrm{CO}_{2}$ and $\mathrm{N}_{2}$ fraction

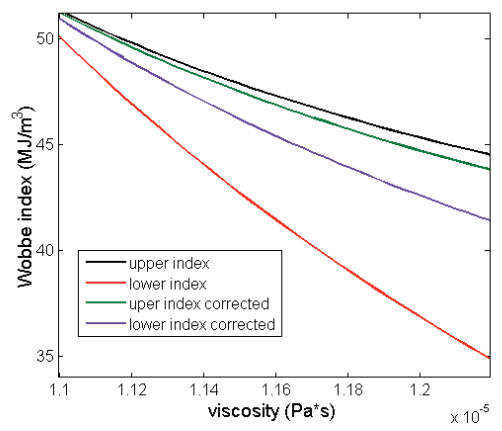

Fig. 4: Determination of the Wobbe index of natural gas [7] from the viscosity, with and without correction for the density

In case $\mathrm{CO}_{2}$ is the only inert gas present in the mixture, there is a strong correlation between the viscosity and the Wobbe index of the gas mixture. However, when also $\mathrm{N}_{2}$ is present, the correlation is less strong due to its higher viscosity, leading to the outer boundaries in figure 4. Since we are able to distinguish between $\mathrm{CO}_{2}$ and $\mathrm{N}_{2}$ by taking the density of the gas mixture into account, we can narrow the band of uncertainty in the Wobbe index considerably to the inner boundaries in figure 4 .

\section{Measurement set-up}

A photograph of the measurement set-up is shown in figure 5 .

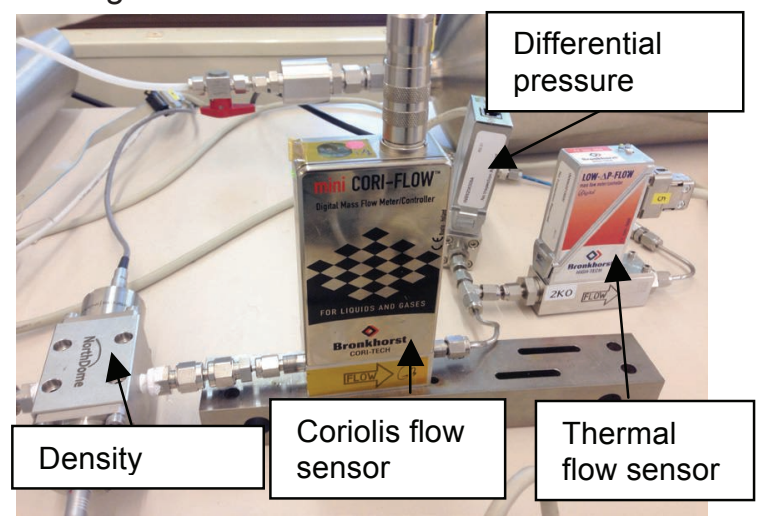

Fig. 5: Measurement set-up 
Methane, propane, carbon dioxide and nitrogen flows in the order of ca. $500 \mathrm{ml} / \mathrm{min}$ were provided to the system, with a pressure in the order of 1.5 bar. During the measurements the output signals of the density, pressure, thermal flow and Coriolis flow sensor were recorded simultaneously, and processed according to the calculation model.

\section{Measurement results and discussion}

When the binary gas mixture $\mathrm{CH}_{4}$ and $\mathrm{CO}_{2}$ is provided to the multi-parameter flow measurement system, the gas composition is determined by a linear equation, based on the heat capacity of the gas mixture, with an accuracy of $1 \%$, as shown in figure 6 .

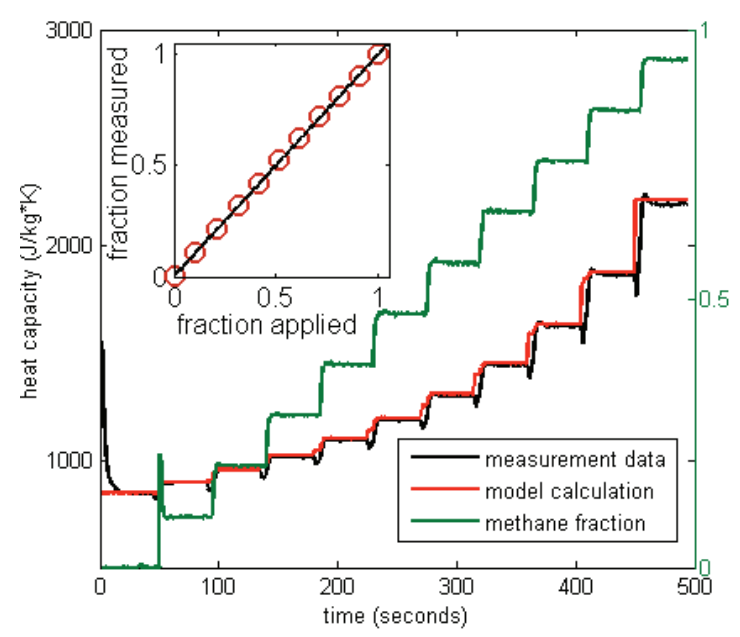

Fig. 6: Composition determination based on heat capacity; modelling and measurement results of a binary gas mixture $\left(\mathrm{CH}_{4} / \mathrm{CO}_{2}\right)$ are within $1 \%$

When the ternary gas mixture $\mathrm{CH}_{4}, \mathrm{CO}_{2}$ and $\mathrm{N}_{2}$ is provided to the system, the composition of the gas mixture is predicted with an accuracy of $5 \%$ by the analytical calculation model, based on the heat capacity and the density of the mixture, as shown in figure 7.

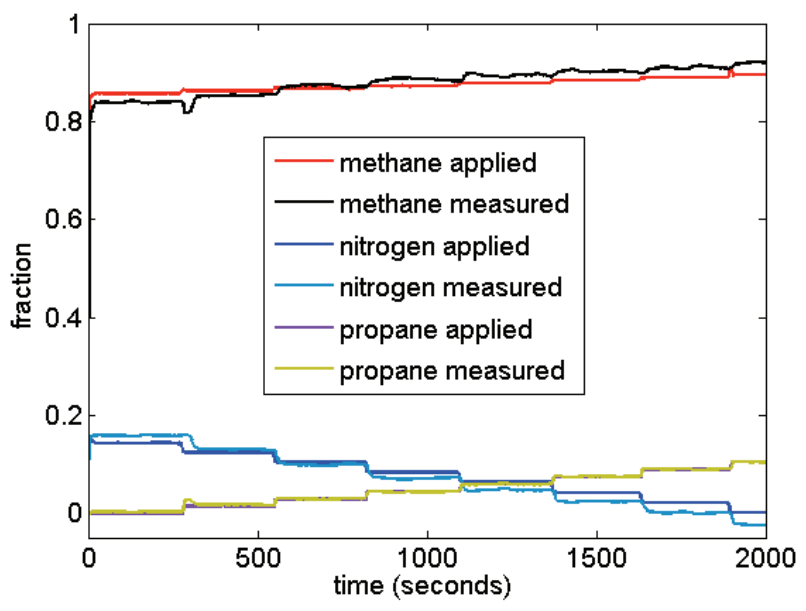

Fig. 7: Composition determination of a ternary gas mixture $\left(\mathrm{CH}_{4} / \mathrm{CO}_{2} / \mathrm{N}_{2}\right)$ based on the heat capacity and density; modelling and measurement results are within $5 \%$.

Since we know the composition and the density, we can calculate the Wobbe index from the ternary gas mixture using (1), as shown in figure 8.

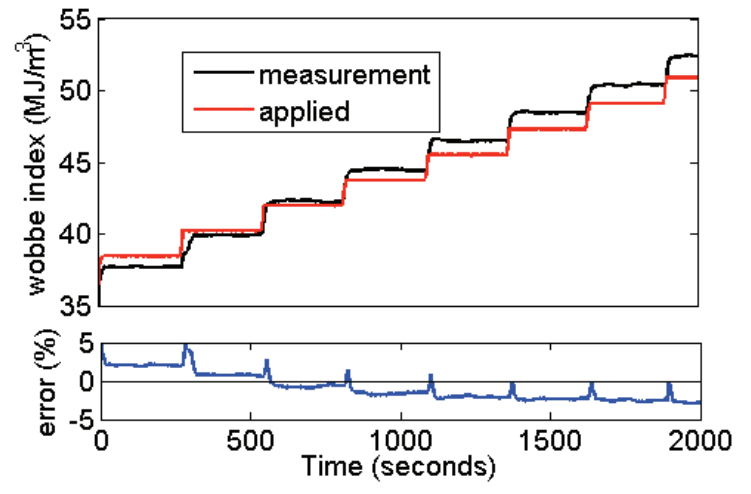

Fig. 8: Wobbe Index determination of a ternary gas mixture $\left(\mathrm{CH}_{4} / \mathrm{CO}_{2} / \mathrm{N}_{2}\right)$; modelling and measurement results are within $5 \%$

When the ternary gas mixture $\mathrm{CH}_{4}, \mathrm{C}_{3} \mathrm{H}_{8}$ and $\mathrm{N}_{2}$ is provided to the system, the gas composition is determined by the analytical calculation model with an accuracy of $5 \%$, based on the heat capacity and the density of the gas mixture, as shown in figure 9. 


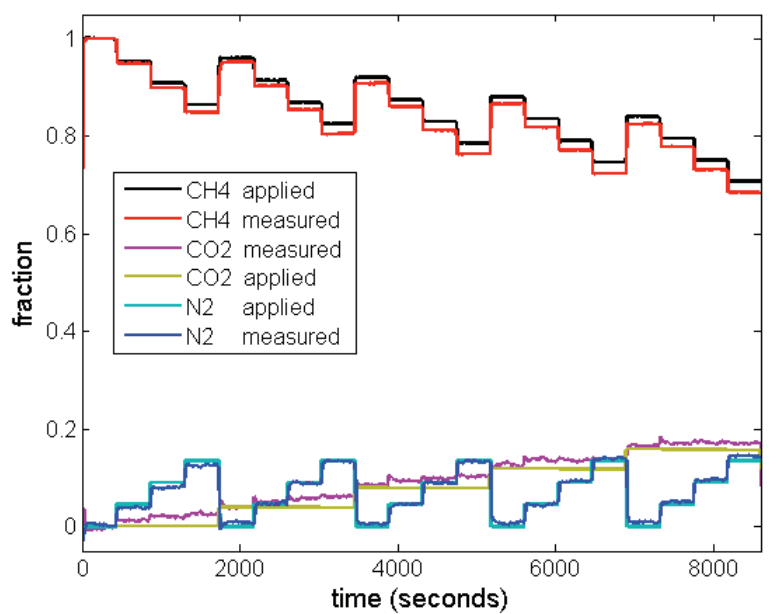

Fig. 9: Composition determination of a ternary gas mixture $\left(\mathrm{CH}_{4} / \mathrm{C}_{3} \mathrm{H}_{8} / \mathrm{N}_{2}\right)$ based on the heat capacity and density; modelling and measurement results are within 5\%

Since we know the composition and the density, we can calculate the Wobbe index from the ternary gas mixture using (1), as shown in figure 10.
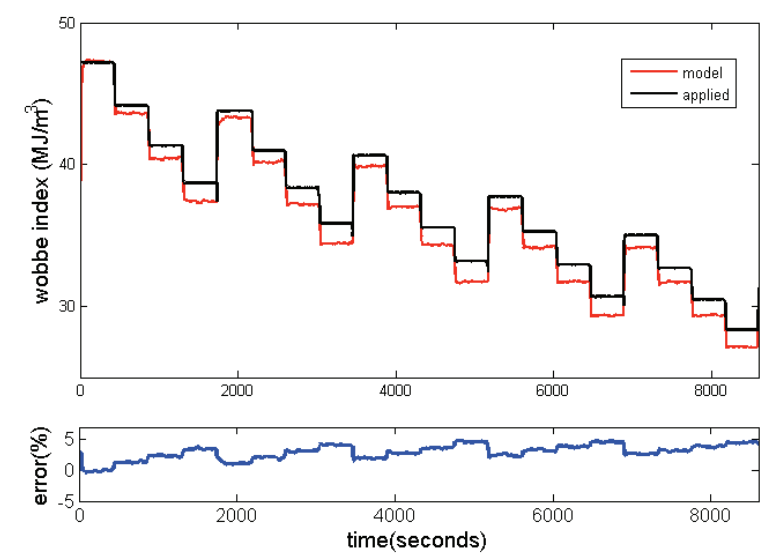

Fig. 10: Wobbe Index determination of a ternary gas mixture $\left(\mathrm{CH}_{4} / \mathrm{C}_{3} \mathrm{H}_{8} / \mathrm{N}_{2}\right)$; modelling and measurement results are within $5 \%$
The deviations mentioned above are caused by a combination of different phenomena. For instance, the system should be carefully flushed such that the adjusted gas mixture is fully established in the entire system. Furthermore, the density sensor has a rather slow response, potentially causing an error in the measured density. Moreover, effects caused by the compressibility of the gas mixture and temperature effects are not taken into account in the model yet. All these issues will be addressed and improved in future work.

\section{Conclusions}

We have designed and realized an analytical calculation model with which we can real-time determine the composition of gas mixtures. The model is based upon a multi-parameter flow measurement system, consisting of a Coriolis and thermal flow sensor, a density meter and a pressure sensor. The system enables direct measurement of flow rate and physical properties of gas mixtures, including density, viscosity and heat capacity. With these parameters we can currently calculate the composition of gas mixtures up to 4 different gases. We demonstrated real-time composition determination of binary $\left(\mathrm{CH}_{4} / \mathrm{N}_{2}\right.$, and $\mathrm{CH}_{4} /$ $\left.\mathrm{CO}_{2}\right)$ and ternary $\left(\mathrm{CH}_{4} / \mathrm{N}_{2} / \mathrm{CO}_{2}\right.$, and $\mathrm{CH}_{4} /$ $\mathrm{C}_{3} \mathrm{H}_{8} / \mathrm{N}_{2}$ ) gas mixtures. For fuel gases, we demonstrated real-time determination of the energy content (Wobbe index) of the gas mixture from its composition and density.

\section{Acknowledgment}

This research was partly financed by the Dutch SBIR and NANONEXT programs. The authors would like to thank the industrial partners in this project for their in-kind contributions and many fruitful discussions. 


\section{References}

[1] A.J. Mouris, Optimized fired heater control, Hydrocarbon Processing, November 2010, pp. 65-68

[2] P. Ulbig, D. Hoburg, Determination of the calorific value of natural gas by different methods, Thermochimica Acta, 382 (2002), pp. 27-35

[3] Lötters, J.C. and Lammerink, T.S.J. and Pap, M.G. and Sanders, R.G.P. and Boer, M.J. de and Mouris, A.J. and Wiegerink, R.J., Integrated micro Wobbe index meter; towards on-chip energy content measurement. In: IEEE 26th International Conference on Micro Electro Mechanical Systems, MEMS 2013, 20-24 January 2013, Taipei, Taiwan (pp. pp. 965-968)

[4] http://www.bright-sensors.com

[5] S. Udina, et al., A micromachined thermoelectric sensor for natural gas analysis: Multivariate calibration results, Sens. Actuators B: Chem. (2012), pp. 551-558

[6] J.C. Lötters, E. van der Wouden, J. Groenesteijn, W. Sparreboom, T.S.J. Lammerink, R.J. Wiegerink, Integrated multiparameter flow measurement system, In: 27th IEEE International Conference on Micro Electro Mechanical Systems, MEMS 2014, 26-30 January 2014, San Francisco, CA, USA (pp. 975-978)

[7] H.B. Levinsky, M.L.D. van Rij, Gaskwaliteit voor de toekomst, Report from KEMA, 27 January 2011 (in Dutch) 